\section{Pneumococcal pneumonia}

\author{
David Goldblatt (1) ,' Elizabeth Miller ${ }^{2}$
}

Pneumococcal conjugate vaccines (PCV) were first licensed in 2000 and have been introduced into national immunisation programme in more than 145 countries. They have had a profound effect on reducing invasive pneumococcal disease (IPD) caused by serotypes in the vaccine. These reductions have been seen both in infants immunised directly with PCV and in the general population protected indirectly by reduced transmission of pneumococci from immunised infants in whom the vaccine prevented nasopharyngeal carriage. PCV7 (Prevenar7, Pfizer, New York, NY, USA) was first introduced in the UK in 2006 followed by PCV13 (Prevenar13, Pfizer, New York, NY, USA) in 2010. Both vaccines have had a striking impact on vaccine serotype IPD although a trend, seen first following PCV7, for an increase in IPD caused by serotypes not included in the vaccine(s) has been reported. ${ }^{1}$ Surveillance of the impact of PCVs is focused on IPD, defined as isolation of pneumococcus from an otherwise sterile site, and has been the bedrock of monitoring the serotype-specific impact of vaccination at a population level for many years. It is however, well understood that from a numerical point of view, IPD is only the tip of the iceberg, with pneumonia and otitis media caused by Streptococcus pneumoniae responsible for a much larger burden of disease.

In this edition of the journal, Pick and colleagues ${ }^{2}$ describe the outcome of their prospective population-based study of adult community acquired pneumonia (CAP) in two teaching hospitals in Nottingham, England. They focus on trends in pneumococcal serotype contribution to the burden of adult CAP over 5 years (2013-2018), a period associated with sustained high coverage of PCV13 in the infant schedule. ${ }^{1}$ Attributing an episode of pneumonia to the pneumococcus is complicated as blood cultures are only positive in approximately $10 \%$ of clinical episodes of pneumonia and the isolation of the pneumococcus from non-sterile sites such as sputum, lacks

${ }^{1}$ Great Ormond Street Institute of Child Health

Biomedical Research Centre, University College London, London, UK

${ }_{2}^{2}$ Infectious Disease Epidemiology, LSHTM, London, UK

Correspondence to Prof David Goldblatt, Profesor David Goldblatt, University College London, London, London, UK; d.goldblatt@ucl.ac.uk specificity for the underlying aetiology of a clinical episode of pneumonia. The ability to detect pneumococcal capsular antigen in the urine of patients with pneumococcal disease has provided a sensitive and specific way of assessing, at the level of serotype, the contribution of the pneumococcus to CAP in adults. Using a recently modified multiplex immunoassay to detect pneumococcal derived carbohydrate antigen in urine ${ }^{3}$ and an assay that detects a common pneumococcal cell wall carbohydrate (C-polysaccharide) the authors were able to evaluate temporal changes in the incidence rate of hospitalised pneumococcal CAP in adults (those over 15 years of age) and the specific contributions of the 24 serotypes measured in their assay.

The authors describe an increase in incidence of CAP overall and pneumococcal CAP over this period, with the latter driven by the increase in nonvaccine pneumococcal CAP and serotype 3 (ST3). Using incident rate ratios (IRRs) the authors reported a statistically significant average annual increase of 19\% over this 5-year period in CAP due to serotypes that are not represented in any currently licensed pneumococcal vaccine, including the 23 valent plain polysaccharide vaccine (PPV23). The only other pneumococcal CAP subgroup that showed a significant increase in IRR over the 5 years was the group of serotypes included in PCV13 and not PCV7 (PCV13non7). This category is however dominated by infection caused by serotype 3 (ST3) which in 2016/2017 and 2017/2018 ST3 represented two-thirds of all PCV13non7 pneumococcal CAP cases.

This dominance of ST3 among the vaccine associated serotypes complicates other associations explored by the authors. For example, the authors attempted to identify potential risk factors in those whose pneumonia is due to serotypes contained in PCV13 compared with other serotypes, which might be informative for a risk based selective PCV13 strategy in adults. A number of risk factors were identified associated with an increased susceptibility to PCV13 serotype disease including increasing age, chronic kidney disease and other risk factors while living in residential care was associated with lower risk. However, none of these associations were significant when ST3 was excluded from the analysis. The preponderance of ST3 among VT pneumococcal
CAP cases in the UK study is consistent with a recent study of CAP in the $\mathrm{US}^{4}$ and with the serotype distribution of IPD cases in both the UK and the USA ${ }^{15}$ and is a consequence of infant PCV13 immunisation having little impact on ST3 carriage. ${ }^{6}$

Since the overall CAP incidence itself irrespective of any vaccine effect may be influenced each year by external factors such as influenza or other respiratory viruses it is informative to look at the percentage of all adult CAP that is caused by vaccine serotypes. PCV7 attributable pneumonia was approximately $2 \%$ of total CAP during this period of study compared with $12.3 \%$ in $2008 / 99^{7}$ soon after PCV7 introduction. PCV13non7 attributable CAP (after exclusion of ST3) accounted for less than $5 \%$ of the total CAP in the last 3 years compared with $10.7 \%$ in 2008/2009. This illustrates the beneficial impact of infant immunisation with PCV on adult VT CAP, at least in settings such as the UK which has achieved high PCV overage over many years. In such settings any additional benefit achieved by immunisation of adults with PCVs containing the same serotypes as those used in infants would be small, although some direct protection in adults against ST3 CAP might be achieved. ${ }^{8}$

One-third of pneumococcal CAP in $2017 / 18$ (34\%) was due to serotypes in the 23 valent vaccine but not in PCV13 (PPV23non13); of these ST8 and ST12F were most prominent, serotypes also shown to be contributing to the increase in IPD in the UK. ${ }^{1}$ A lack of consistent evidence showing the effectiveness of PPV23 against CAP $^{9}$ has led to PPV23non13 serotypes included in new extended multivalent pneumococcal conjugate vaccine being evaluated. Following licensure, the use of extended valency vaccines will be influenced by a number of factors including their licensed indication (IPD or both IPD and pneumonia), the age group for which they are licensed (adults only or adults and children), their serotype composition and their likely cost effectiveness, bearing in mind that they will be licensed on immunogenicity alone, and at the time of licensure there will be no direct evidence for the ability of the vaccines to prevent CAP caused by the novel serotypes contained therein.

The limitation of evaluating urine with an assay that can only recognise 24 antigens is apparent from the large increase in serotypes referred to by the authors as untyped. These episodes of pneumonia were associated with a positive C-polysaccharide urinary antigen detection test but no confirmatory 24 valent bioplex results. 
The study therefor fails to provide comprehensive information about emerging pneumococcal strains which are not included in licensed vaccines. Furthermore, some of the monoclonal antibodies cross react with more than one pneumococcal capsule and for 11 of the 24 serotypes detected in the urine, the authors have been unable to categorically assign a serotype and have relied on a probabilistic technique based on dominant serotypes in IPD. This is based on the assumption that a serotype causing pneumonia has a similar propensity for causing IPD which may not be the case. In particular, for two serotypes assigned causality in this way, $11 \mathrm{~A}$ and $15 \mathrm{~A}$, there may be significant contributions from $16 \mathrm{~F}$ and $15 \mathrm{~B} / \mathrm{C}$, respectively.

Pick and colleagues ${ }^{2}$ provide useful information on trends in adult pneumonia over the last 5 years and the effect of PCV vaccination on the proportion that is pneumococcal-attributable. Their analysis has shed light on the increase in adult hospital admissions due to pneumonia in recent years ${ }^{10}$ showing that this is attributable in part to pneumococcal serotype replacement. The observation that the majority of adult pneumonia secondary to serotypes effective in infant vaccines is reduced is reassuring and reinforces the need for maintaining high infant coverage. It also demonstrates that providing the same PCV as used in infants to adults is unlikely to have a big impact on CAP, an observation noted in the USA recently which led to the reversal of the former recommendation in the USA to use PCV in all adults over 65 years of age. ${ }^{11}$ New conjugate vaccines with wider serotype coverage are in development but without a better understanding of the drivers of serotype replacement, especially among the elderly, their overall impact on pneumococcal disease in the future remains uncertain.

Funding The authors have not declared a specific grant for this research from any funding agency in the public, commercial or not-for-profit sectors.

Competing interests DG's laboratory receives grant funding from industry partners including the vaccine manufacturers GSK, Merck and Sanofi Pasteur. DG receives occasional honoraria from industry partners for participation in advisory boards.

Patient consent for publication Not required.

Provenance and peer review Commissioned; internally peer reviewed.

\section{(2) OPEN ACCESS}

Open access This is an open access article distributed in accordance with the Creative Commons Attribution Non Commercial (CC BY-NC 4.0) license, which permits others to distribute, remix, adapt, build upon this work non-commercially, and license their derivative works on different terms, provided the original work is properly cited, appropriate credit is given, any changes made indicated, and the use is non-commercial. See: http:// creativecommons.org/licenses/by-nc/4.0/.

(c) Author(s) (or their employer(s)) 2020. Re-use permitted under CC BY-NC. No commercial re-use. See rights and permissions. Published by BMJ.

Check for updates

To cite Goldblatt D, Miller E. Thorax 2020;75:6-7.

Accepted 26 October 2019

Published Online First 21 November 2019

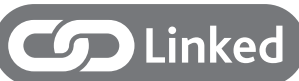

- http://dx.doi.org/10.1136/thoraxjnl-2019-213725

Thorax 2020;75:6-7.

doi:10.1136/thoraxjnl-2019-214135

ORCID iD

David Goldblatt http://orcid.org/0000-0002-0769-5242

\section{REFERENCES}

1 Ladhani SN, Collins S, Djennad A, et al. Rapid increase in non-vaccine serotypes causing invasive pneumococcal disease in England and Wales, 2000-17: a prospective national observational cohort study. Lancet Infect Dis 2018;18:441-51.

2 Pick H, Daniel P, Rodrigo C, et al. Pneumococcal serotype trends, surveillance and risk factors in UK adult pneumonia, 2013-18. Thorax 2020;75:37-48.

3 Eletu SD, Sheppard CL, Thomas E, et al. Development of an Extended-Specificity multiplex immunoassay for detection of Streptococcus pneumoniae serotype-specific antigen in urine by use of human monoclonal antibodies. Clin. Vaccine Immunol. 2017;24

4 McLaughlin JM, Jiang Q, Isturiz RE, et al. Effectiveness of 13-Valent pneumococcal conjugate vaccine against hospitalization for community-acquired pneumonia in older us adults: a test-negative design. Clin Infect Dis 2018:67:1498-506.

5 Pilishvili T. Impact of PCV13 on invasive pneumococcal disease (IPD) burden and the serotype distribution in the US Advisory Committee on immunization practices: OCT 24, 2018. Available: https://www.cdc.gov/ vaccines/acip/meetings/downloads/slides-2018-10/ Pneumococcal-02-Pilishvili-508.pdf

6 Dagan R, Patterson S, Juergens C, et al. Comparative immunogenicity and efficacy of 13-valent and 7-valent pneumococcal conjugate vaccines in reducing nasopharyngeal colonization: a randomized doubleblind trial. Clin Infect Dis 2013;57:952-62.

7 Rodrigo C, Bewick T, Sheppard C, et al. Impact of infant 13-valent pneumococcal conjugate vaccine on serotypes in adult pneumonia. Eur Respir $J$ 2015;45:1632-41.

8 Gessner BD, Jiang Q, Van Werkhoven $\mathrm{CH}$, et al. A post-hoc analysis of serotype-specific vaccine efficacy of 13-valent pneumococcal conjugate vaccine against clinical community acquired pneumonia from a randomized clinical trial in the Netherlands. Vaccine 2019:37:4147-54.

9 Tin Tin Htar M, Stuurman AL, Ferreira G, et al. Effectiveness of pneumococcal vaccines in preventing pneumonia in adults, a systematic review and meta-analyses of observational studies. PLoS One 2017;12:e0177985.

10 Thorrington D, Andrews N, Stowe J, et al. Elucidating the impact of the pneumococcal conjugate vaccine programme on pneumonia, sepsis and otitis media hospital admissions in England using a composite control. BMC Med 2018:16:13.

11 CDC, 2019. Available: https://www.cdc.gov/vaccines/ acip/recommendations.htm 